\title{
Photoluminescence and free carrier interactions in erbium-doped GaAs
}

\author{
T. D. Culp, J. G. Cederberg, B. Bieg, T. F. Kuech, and K. L. Bray a) \\ Department of Chemical Engineering, University of Wisconsin, Madison, Wisconsin 53706 \\ D. Pfeiffer and C. H. Winter \\ Department of Chemistry, Wayne State University, Detroit, Michigan 48202
}

(Received 12 November 1997; accepted for publication 27 January 1998)

\begin{abstract}
The photoluminescence properties of GaAs:Er doped with a new pyrazole and pyridine-based Er source, tris(3,5-di-tert-butylpyrazolato)bis(4-tert-butylpyridine)erbium(III), were investigated. These samples showed significantly stronger and sharper $1.54 \mu \mathrm{m} \mathrm{Er}^{3+}$ luminescence than in GaAs:Er samples doped with cyclopentadienyl-based Er sources. The efficient luminescence was associated with the $\mathrm{Er}-2 \mathrm{O}$ center, formed with unintentional oxygen impurities. $\mathrm{The}^{3{ }^{3+}}$ emission was greatly reduced in $n$-type samples, whereas the emission remained strong in $p$-type samples. This trend suggests that either the free hole concentration is very important to the $\mathrm{Er}^{3+}$ excitation efficiency, and/or there is a strong Auger quenching mechanism which involves free electrons. A model based on the results of a two-beam experiment indicates the presence of strong Auger energy transfer from the Er-bound exciton to a free electron. Auger energy transfer from the excited $\mathrm{Er}^{3+}$ ion to a free electron was found to be much less important. The temperature dependence of the $\mathrm{Er}^{3+}$ emission was also examined. A decrease in intensity was observed at the lowest temperatures. This effect was attributed to the freeze-out of carriers onto a relatively shallow trap which could be related to either Er or shallow acceptors. (C) 1998 American Institute of Physics.
\end{abstract}

[S0021-8979(98)02809-6]

\section{INTRODUCTION}

Rare-earth doped semiconductors have gained significant attention in recent years as a promising new class of electrically activated infrared-emitting materials. ${ }^{1,2}$ The intra- $4 f$ transitions of rare-earth ions give rise to sharp emission lines whose wavelengths are largely independent of both the host material and temperature. This stability occurs because the filled outer $5 s$ and $5 p$ electron shells screen transitions within the inner $4 f$ electron shell from interaction with the host. The ${ }^{4} I_{13 / 2} \rightarrow{ }^{4} I_{15 / 2}$ emission of $\mathrm{Er}^{3+}$ at $1.54 \mu \mathrm{m}$ is of special interest because this wavelength matches the minimum loss region of silica fibers used in optical communications.

The practicality of these materials depends on the efficiency of rare-earth luminescence, which, in turn, depends on the interaction of the rare-earth ion with the semiconductor host and the mechanisms of rare-earth excitation and deactivation. At the moment, a full understanding of the properties of rare-earth dopants in semiconductors and their relationship to growth processes is incomplete.

In this work, we have examined the photoluminescence (PL) properties of GaAs:Er doped using a new pyrazole and pyridine-based Er precursor. Comparisons will be made with our previous results on GaAs:Er samples grown with cyclopentadienyl-based sources. ${ }^{3,4}$ The new samples exhibited strong, sharp luminescence despite low Er concentrations. However, we found that the $\mathrm{Er}^{3+}$ emission was

\footnotetext{
a) Author to whom correspondence should be addressed. Current address: Department of Chemistry, Washington State University, Pullman, WA 99164.
}

strongly quenched in $n$-type samples. Several possible mechanisms for this quenching are discussed. Furthermore, the results of a two-beam experiment and a detailed model are presented, which indicate the presence of Auger energytransfer processes involving free electrons. Finally, the temperature dependence of the $\mathrm{Er}^{3+}$ emission is discussed, including an effect associated with carrier freeze-out at low temperatures.

\section{EXPERIMENT}

Epitaxial GaAs films were prepared by metalorganic vapor phase epitaxy (MOVPE) in a horizontal, low-pressure (78 Torr) reactor using $\left(\mathrm{CH}_{3}\right)_{3} \mathrm{Ga}$ and $\mathrm{AsH}_{3}$. Palladium diffused $\mathrm{H}_{2}$ was used as the carrier gas. Layers nominally $3 \mu \mathrm{m}$ thick were deposited on semi-insulating (100) oriented GaAs substrates. Erbium doping was accomplished using tris(3,5di-tert-butylpyrazolato)bis(4-tert-butylpyridine)erbium(III).

The synthesis and doping characteristics of the erbium precursor are reported elsewhere. ${ }^{5,6}$ For the photoluminescence studies reported here, all samples were grown at $600{ }^{\circ} \mathrm{C}$ with a V/III ratio of 60 .

In the PL measurements, samples were cooled in a variable temperature cryostat. Excitation was performed with the $514.5 \mathrm{~nm}$ line of a chopped argon ion laser at an intensity of approximately $3 \mathrm{~W} / \mathrm{cm}^{2}$. Luminescence was collected and dispersed with a $1 \mathrm{~m}$ monochromator. In measuring the PL spectra, detection was with a liquid-nitrogen cooled Ge detector using standard lock-in amplifier techniques. All spectra were corrected for instrumental response. For lifetime measurements, a faster Ge detector with a response time of 


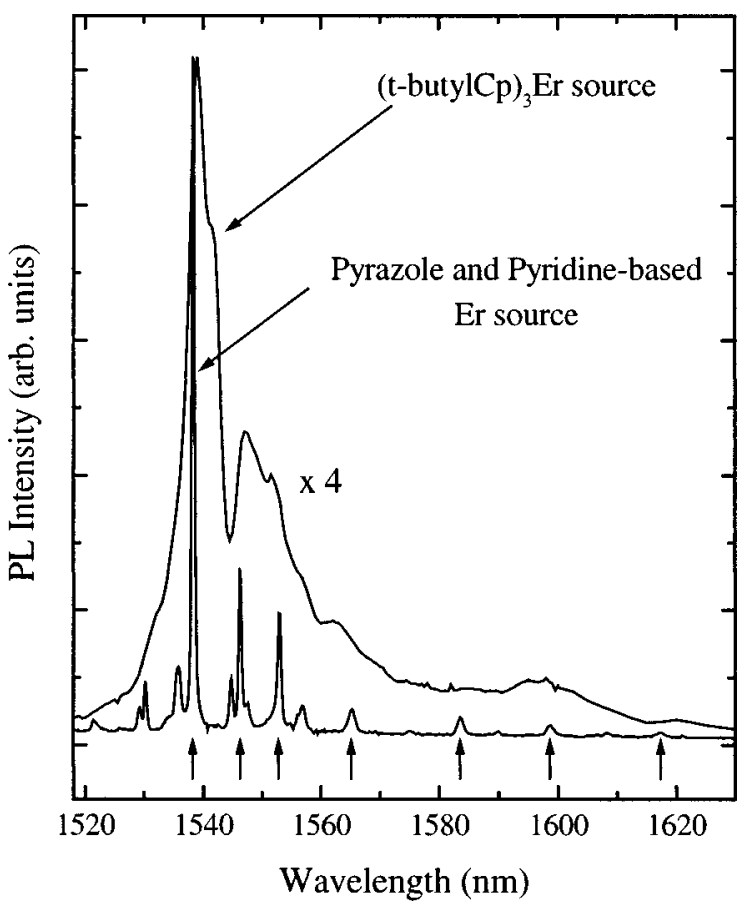

FIG. 1. PL spectra comparing the ${ }^{4} I_{13 / 2} \rightarrow{ }^{4} I_{15 / 2} \mathrm{Er}^{3+}$ emission in GaAs:Er samples doped with ( $t$-butyl $\mathrm{Cp})_{3} \mathrm{Er}$ and (3,5-di- $t$-butylpyrazolato $)_{3}(4-$ $t$-butylpyridine $)_{2}$ Er. Arrows indicate peaks which match the seven main emission lines of the Er-2O center (see Ref. 7). The measurement temperature was $12 \mathrm{~K}$, and the instrument resolution was $0.4 \mathrm{~nm}$.

$\sim 180 \mu$ s was used, and the signal was analyzed with a digital oscilloscope.

\section{RESULTS AND DISCUSSION}

\section{A. Photoluminescence of the $\mathrm{Er}-2 \mathrm{O}$ center}

In Fig. 1, the ${ }^{4} I_{13 / 2} \rightarrow{ }^{4} I_{15 / 2} \mathrm{Er}^{3+}$ emission from GaAs:Er samples doped with the pyrazole and pyridine-based Er source is compared with the emission from previous GaAs:Er samples grown using ( $t$-butyl $\mathrm{Cp}$ ) ${ }_{3}$ Er. Samples doped with another cyclopentadienyl-based $\mathrm{Er}$ source, ( $i$-propyl $\mathrm{Cp})_{2} \mathrm{CpEr}$, showed a nearly identical spectrum as the latter. With the pyrazole and pyridine-based Er precursor, there is a drastic reduction in the main peak linewidth, as well as a significant increase in the peak intensity. The integrated intensity is roughly the same. One explanation is that the pyrazole and pyridine-based Er source produces a very well-defined $\mathrm{Er}^{3+}$ center, which leads to a small linewidth and high peak intensity. The main peak linewidth was limited by the equipment resolution, but is less than $0.7 \mathrm{~cm}^{-1}$. On the other hand, the cyclopentadienyl-based Er sources produce a wide distribution of $\mathrm{Er}^{3+}$ centers in slightly different environments, leading to a broader linewidth of approximately $40 \mathrm{~cm}^{-1}$.

Many sharp, intense peaks are observed in the spectrum of the GaAs:Er sample doped with the pyrazole and pyridine-based source. In fact, the seven peaks indicated by arrows exactly match the main emission peaks of the $\mathrm{Er}-2 \mathrm{O}$ center identified by Takahei et al. ${ }^{7} \mathrm{~A}$ wide range of techniques have been used to characterize this center, including Rutherford backscattering (RBS), ${ }^{8}$ secondary ion mass spec- troscopy (SIMS), ${ }^{7,8}$ Zeeman analysis, ${ }^{9}$ polarized photoluminescence, ${ }^{10}$ and photoluminescence excitation (PLE) ${ }^{10-12}$ spectroscopy, and PL studies of samples alloyed with $1 \%$ aluminum. ${ }^{8}$ As a result, this center is known to possess $C_{2 V}$ symmetry, and the microscopic structure is believed to be an erbium atom on a Ga site with two nearest neighbor oxygen atoms, either on As sites or on tetrahedral interstitial sites. The well-defined structure of this center leads to the narrow peak linewidths. Indeed, Takahei and co-workers report a main peak linewidth of less than $0.1 \mathrm{~cm}^{-1}{ }^{13}$

To produce this Er-2O center, Takahei et al. intentionally introduced a few ppm $\mathrm{O}_{2}$ gas into the growth chamber. However, in this study, the Er-2O center was observed despite the fact that no such intentional oxygen source was used. Although unintentional oxygen could arise from many sources, the oxygen is not believed to originate in the erbium precursor. Oxygen impurities in the erbium source would most likely come from adventitious water. However, water would cause a pyrazolato ligand to be expelled from the source compound, leading to a much less volatile or nonvolatile compound. Another possibility is contamination of the source compound with tetrahydrofuran, traces of which might be present in the dry box that was used in the source preparation. However, tris(3,5-di-tert-butylpyrazolato) bis(tetrahydrofuran)erbium(III) decomposes upon sublimation attempts. ${ }^{5}$ Furthermore, the ${ }^{1} \mathrm{H}$ NMR spectrum of the yttrium analog of the Er precursor showed no signals attributable to tetrahydrofuran, or any other ligand except those expected. ${ }^{5}\left({ }^{1} \mathrm{H}\right.$ NMR cannot be used on the Er compound itself because $\mathrm{Er}^{3+}$ is paramagnetic.) As a result, it is believed that the erbium compound sublimes in a very pure state.

There are several other possible sources of oxygen in the MOVPE system. The most likely source is trace amounts of metal alkoxides in the metalorganic sources. Also, trace amounts of $\mathrm{H}_{2} \mathrm{O}$ could be introduced during the sample loading procedure. However, the oxygen and water concentrations are estimated to be several orders of magnitude below the ppm level intentionally introduced by Takahei et al. It appears that the new pyrazole and pyridine-based precursor is very efficient at scavenging even small concentrations of oxygen impurities, incorporating them into the layer in the form of the $\mathrm{Er}-2 \mathrm{O}$ center.

The other sharp peaks in Fig. 1 are due to transitions from higher lying states in the ${ }^{4} I_{13 / 2}$ manifold. These upper states become populated from a small amount of laser heating, as evidenced by the fact that the relative intensities of these extra peaks decreased at lower excitation powers. Also, the relative intensities of these peaks increased at higher temperatures. Figure 2 shows these "hot lines" in more detail, along with their spacings from the seven main peaks. From these spacings, the next two highest levels in the ${ }^{4} I_{13 / 2}$ manifold were found to be about 4.2 and $8.9 \mathrm{meV}$ above the lowest state.

\section{B. Physical and electrical characterization}

SIMS analysis was performed on selected samples to determine erbium and oxygen concentrations. However, both 


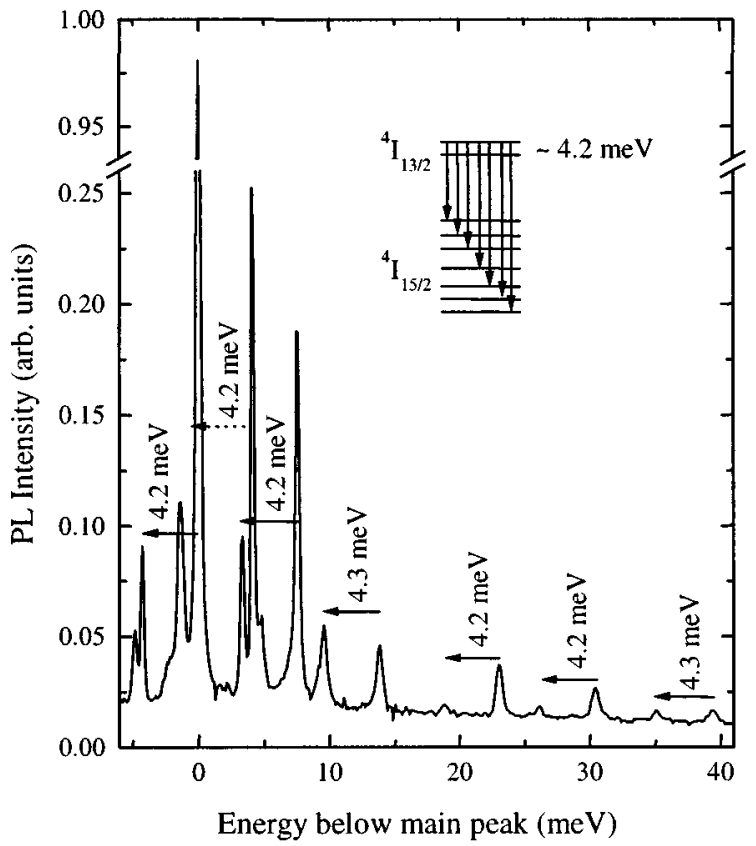

(a)

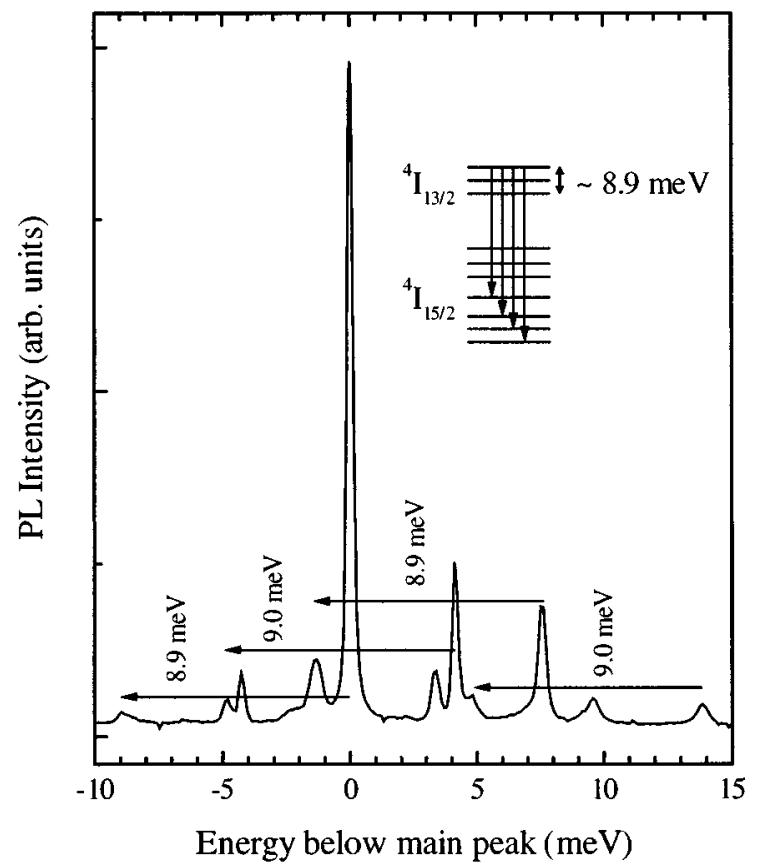

(b)

FIG. 2. PL spectra indicating hot line transitions from the (a) second and (b) third levels of the ${ }^{4} I_{13 / 2} \mathrm{Er}^{3+}$ manifold to the states of the ${ }^{4} I_{15 / 2}$ manifold. Energy shifts from the main peaks (transitions from the lowest level of the ${ }^{4} I_{13 / 2}$ manifold) are indicated. Note that the dotted arrow in part (a) is not indicating the main peak. Rather, it is pointing to another peak obscured under the main peak. The measurement temperature was $12 \mathrm{~K}$, but the hot lines were induced by a small amount of laser heating (power density $\sim 3 \mathrm{~W} / \mathrm{cm}^{2}$ ). The measurement resolution is $\sim 0.2 \mathrm{meV}$.

the Er and O concentrations were below the SIMS detection limits of $3 \times 10^{16}$ and $4 \times 10^{17} \mathrm{~cm}^{-3}$, respectively. Capacitance-voltage electrochemical profiling measurements were also performed on samples co-doped with Er and

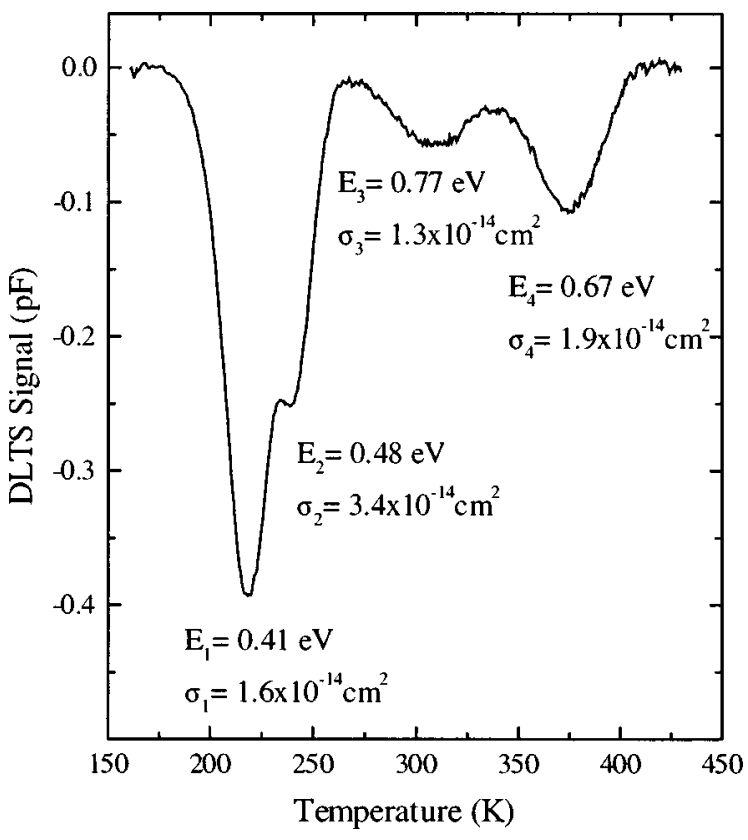

FIG. 3. DLTS spectrum of GaAs:Er,Si at a rate window of $46.5 \mathrm{~s}^{-1}$. The Si concentration was $\sim 7 \times 10^{16} \mathrm{~cm}^{-3}$. Electron trap energies and capture cross sections are indicated. The third trap exhibited an electric field dependence, with the zero-field activation energy reported in the figure (see Ref. 6). The trap concentrations ranged from $10^{13}$ to $10^{14} \mathrm{~cm}^{-3}$.

shallow impurities, although these measurements can only give the concentration of electrically active deep levels due to erbium incorporation, not the actual physical erbium concentration. Samples co-doped with $\mathrm{Si}$ showed free carrier compensation of approximately $10^{16} \mathrm{~cm}^{-3}$ due to erbium doping. The dependence of the erbium-related compensation upon growth parameters is discussed elsewhere. ${ }^{6}$

Based upon these results, the erbium concentration in these samples is no higher than $\sim 10^{16} \mathrm{~cm}^{-3}$. Samples grown under similar conditions but doped with the cyclopentadienyl-based Er precursors showed much higher erbium concentrations ranging from $10^{17}-10^{18} \mathrm{~cm}^{-3}$ as determined by SIMS. ${ }^{3}$ The electrically active Er concentration in these samples was about $2 \times 10^{17} \mathrm{~cm}^{-3}$. However, despite the much lower concentration in the samples doped with the pyrazole and pyridine-based source, the integrated Er PL intensity is roughly the same, and the peak intensity is much stronger. This indicates that the erbium is introduced as a very efficient optical center.

Preliminary deep level transient spectroscopy (DLTS) studies were also performed on $\mathrm{GaAs}: \mathrm{Er}, \mathrm{Si}$ and presented separately. ${ }^{6}$ One typical DLTS spectrum is shown in Fig. 3. For each peak, the corresponding trap energy and capture cross section are also indicated, as determined from an Arrhenius plot. A series of electron traps are observed from 0.41 to $0.77 \mathrm{eV}$ below the conduction band. In contrast, the samples doped with the cyclopentadienyl-based Er sources showed hole traps 0.54 and $0.62 \mathrm{eV}$ above the valence band. Another interesting distinction is observed in the capture cross sections for these traps. The capture cross sections for the electron traps in the samples doped with the pyrazole and pyridine-based source are on the order of $10^{-14} \mathrm{~cm}^{2}$. This is one to two orders of magnitude larger than the capture cross 


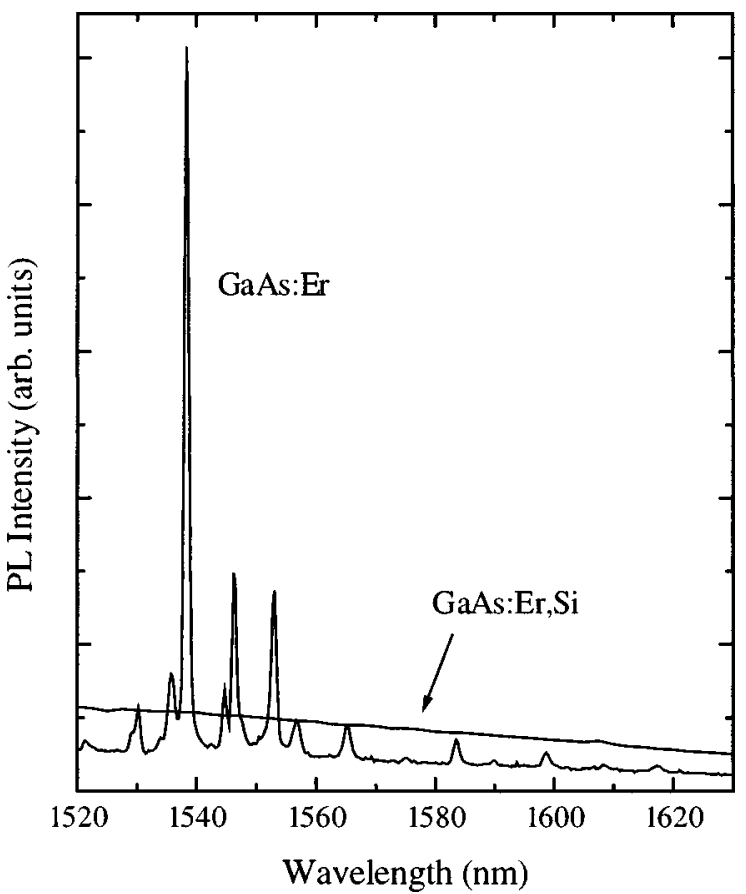

FIG. 4. PL spectra around $1.54 \mu \mathrm{m}$ for GaAs:Er and GaAs:Er,Si. The measurement temperature was $12 \mathrm{~K}$.

sections for the hole traps in the samples doped with the cyclopentadienyl-based $\mathrm{Er}$ sources $\left(8.7 \times 10^{-16}\right.$ and 1.6 $\times 10^{-16} \mathrm{~cm}^{2}$, respectively). This large difference in capture cross sections leads to a tempting explanation for the differences in PL intensities. If these traps are the Er-related traps which lead to $\mathrm{Er}^{3+}$ excitation and emission, the larger capture cross section might explain the efficient photoluminescence despite the low Er concentration in the samples grown with the pyrazole and pyridine-based source. However, as is discussed in the next section, co-doping with shallow impurities adds a complicating factor which makes this speculation less certain.

\section{PL characteristics of samples with shallow co-dopants}

GaAs:Er samples showed $p$-type conductivity with a hole concentration of $\sim 2 \times 10^{15} \mathrm{~cm}^{-3}$, as determined by Van der Pauw-Hall measurement. The $p$-type conductivity most likely resulted from carbon incorporation during growth. For the $C-V$ profiling and DLTS electrical measurements, GaAs:Er samples were co-doped with $\mathrm{Si}$ to provide excess free electrons $\left(n \sim 7 \times 10^{16} \mathrm{~cm}^{-3}\right.$ at room temperature). However, it must be assessed whether this co-doping itself affects the nature of the $\mathrm{Er}^{3+}$ centers. Indeed, Fig. 4 shows that co-doping with $\mathrm{Si}$ completely inhibited the $\mathrm{Er}^{3+}$ luminescence. Several explanations are possible for this behavior. First, the same $\mathrm{Er}^{3+}$ center was present in the codoped samples, but no emission was observed due to the decreased minority carrier lifetime (increased band edge recombination rate) associated with shallow impurity doping. In other words, competition for free carriers by other recombination pathways was greatly increased at the expense of the Er excitation pathway. Second, the same $\mathrm{Er}^{3+}$ center was

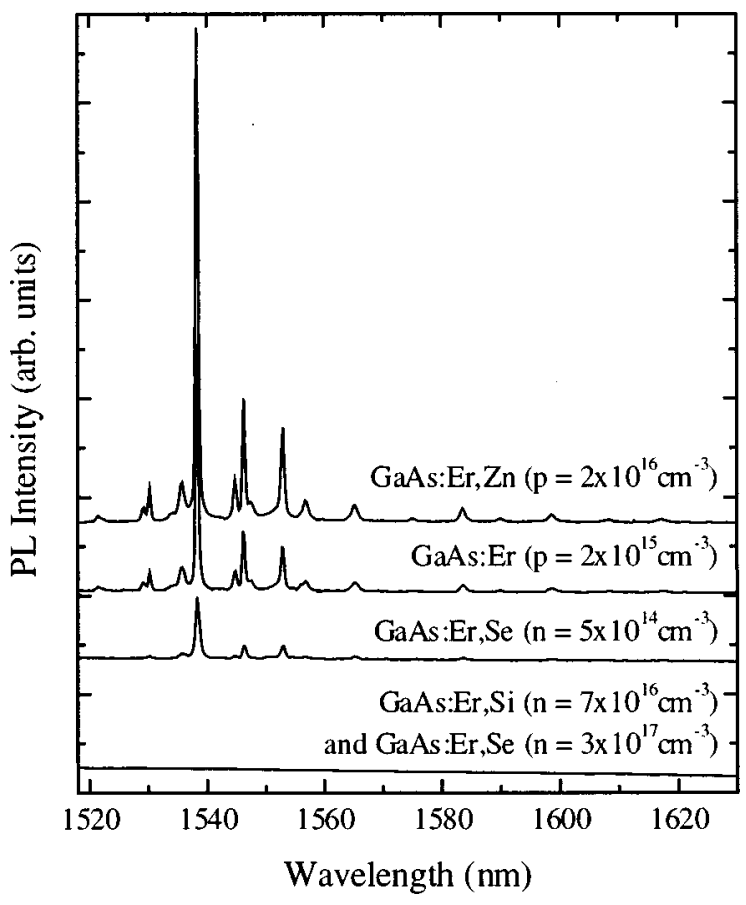

FIG. 5. PL spectra comparing the $\mathrm{Er}^{3+}$ emission of GaAs:Er samples codoped with $\mathrm{Zn}$, Se, or $\mathrm{Si}$. The room temperature carrier concentration and type are indicated, as determined from Hall measurements. The PL spectra were obtained at $12 \mathrm{~K}$.

present, but no emission was observed due to some Fermilevel effect as the Si doping shifted the Fermi level towards the conduction band. Finally, the same $\mathrm{Er}^{3+}$ center may not have been present at all. It is possible that nonoptically active $\mathrm{Er}-\mathrm{Si}$ complexes were formed.

To help distinguish between these possibilities, samples were co-doped with other shallow dopants. First, $p$-type GaAs:Er,Zn samples were grown. These samples showed room temperature hole concentrations of $\sim 2 \times 10^{16} \mathrm{~cm}^{-3}$. As shown in Fig. 5, the GaAs:Er,Zn samples exhibited the identical $\mathrm{Er}-2 \mathrm{O}$ spectrum, and in fact, the intensity was approximately two times stronger than in GaAs:Er. This result indicates that the lack of $\mathrm{Er}^{3+}$ emission from the $\mathrm{Si}$ co-doped samples was not just an effect of the decreased minority carrier lifetime and increased competition for free carriers by other recombination pathways, as the $\mathrm{Zn}$ co-doped samples have a similarly reduced minority carrier lifetime.

Next, $n$-type samples with a different donor, Se, were grown. Samples with room temperature electron concentrations of $3 \times 10^{17}$ and $1 \times 10^{18} \mathrm{~cm}^{-3}$ showed no $\mathrm{Er}^{3+}$ emission, similar to the Si co-doped samples. A more lightly $\mathrm{Se}$ co-doped sample with an electron concentration of $\sim 5$ $\times 10^{14} \mathrm{~cm}^{-3}$ was also examined. In this case, the identical Er-2O spectrum was again observed, as shown in Fig. 5, although the intensity was about a factor of 4 weaker than in GaAs:Er. The weak emission in the lightly Se co-doped sample and the lack of emission in the more heavily Se and $\mathrm{Si}$ co-doped samples may indicate that a nonoptically active Er-donor complex was formed. However, we believe this explanation is unlikely. It is doubtful that the same type of complex would form in both materials, since Si prefers a $\mathrm{Ga}$ site, whereas Se prefers an As site. The hypothetical Er- 
donor complex would have to provide an energetic driving force for pulling Si onto As sites at low concentrations, making the $\mathrm{Si}$ and $\mathrm{Se}$ cases analogous.

Therefore, the most likely explanation for the trend in intensity between the co-doped materials (Fig. 5) is that the shift of the Fermi level towards the conduction band inhibits $\mathrm{Er}^{3+}$ luminescence. This effect could indicate a strong dependence of the $\mathrm{Er}^{3+}$ excitation efficiency upon free hole density. Conversely, a strong quenching mechanism involving free electrons could be present. These possibilities are not mutually exclusive, and both could be independently important. A third explanation is that shifting the Fermi level towards the conduction band promotes the creation of gallium vacancies, which may interact with $\mathrm{Er}$ atoms and prevent formation of the $\mathrm{Er}-2 \mathrm{O}$ center. This possibility has been discussed in more detail in a separate article. ${ }^{6}$ Finally, it is possible, although unlikely, that the shift of the Fermi level changes the overall charge state of the Er-2O complex such that the complex is no longer optically active. The first two explanations, involving the importance of free holes and free electrons, are discussed below.

$\mathrm{Er}^{3+}$ excitation is believed to occur according to the following model, first developed for InP:Yb. ${ }^{14,15}$ In this model, the rare-earth ion introduces either an electron trap below the conduction band or a hole trap above the valence band. A photogenerated electron or hole is trapped at the rare-earth center, which then attracts an opposite carrier through its long-range Coulombic potential. This bound exciton then recombines and transfers its energy to the $4 f$ shell of the rareearth ion.

Studies of the thermal quenching of the $\mathrm{Er}^{3+}$ emission in $\mathrm{GaAs}: \mathrm{Er}^{4}$ and $\mathrm{GaAs}: \mathrm{Er}, \mathrm{O}^{16}$ under hydrostatic pressure gave tentative evidence that $\mathrm{Er}^{3+}$ introduced an electron trap below the conduction band rather than a hole trap above the valence band. In this case, thermal dissociation of the bound exciton through hole emission was found to be an important quenching mechanism, as the hole had a binding energy of only $\sim 13 \mathrm{meV}^{4}$ Thus, to have efficient $\mathrm{Er}^{3+}$ excitation, it may be necessary to have a large surplus of free holes to overcome the hole emission rate and drive the formation of bound excitons. Optical pumping only creates a small number of free holes $\left(\sim 10^{11} \mathrm{~cm}^{-3}\right.$ as estimated from the model presented below). Therefore, to provide a hole concentration larger than the $\mathrm{Er}^{3+}$ concentration, shallow acceptor doping is needed. This effect could explain the much stronger intensity observed in the $p$-type samples (GaAs:Er, GaAs:Er,Zn) as compared to the $n$-type samples (GaAs:Er,Se, GaAs:Er,Si).

In addition to the importance of free holes in $\mathrm{Er}^{3+}$ excitation, there could also be quenching mechanisms which involve free electrons. Specifically, two types of nonradiative Auger transitions involving excess free carriers could exist. In the first mechanism, proposed for $\mathrm{Si}^{-\mathrm{Er}^{17-19}}$ and $\mathrm{CdF}_{2}: \mathrm{Gd}^{20,21}$ the excited rare-earth ion decays and transfers its energy to a free carrier rather than emitting a photon. In the second mechanism, proposed for $\mathrm{GaAs}: \mathrm{Er}^{22,23}$ and InP:Er, ${ }^{24}$ the bound exciton localized on the rare-earth ion recombines and transfers its energy to a free carrier rather than to the rare-earth ion. In both mechanisms, the excited

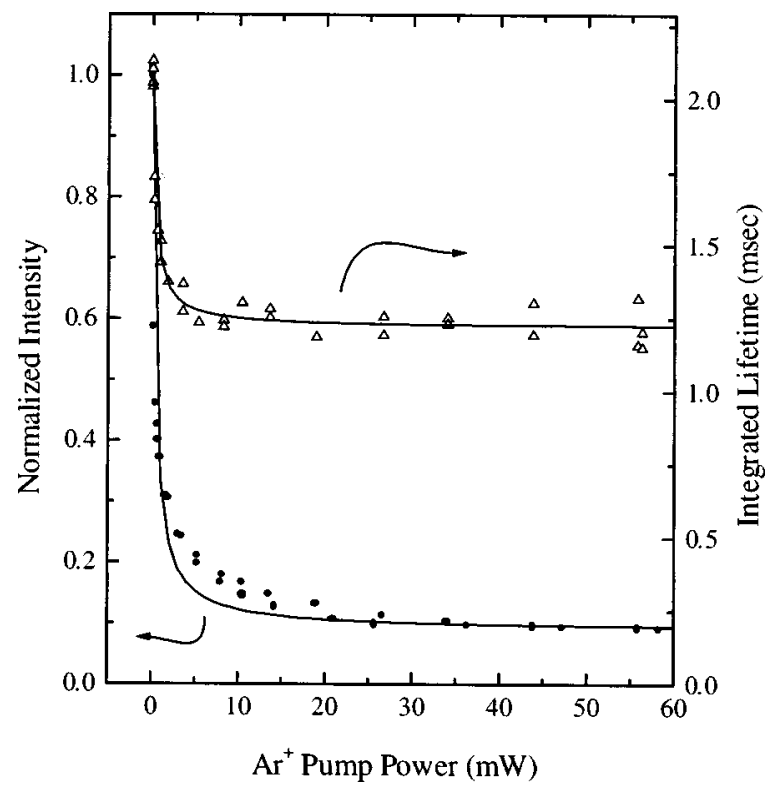

FIG. 6. Modulated PL intensity and lifetime at $1538.3 \mathrm{~nm}$ in the two-beam experiment as a function of the unchopped laser excitation power. The lines are fit to the model presented in the text. The measurement temperature was $12 \mathrm{~K}$.

free carrier subsequently thermalizes to the band edge through phonon emission.

Either of these mechanisms can explain the decrease in intensity observed in $n$-type samples, if the Auger process preferentially couples to free electrons rather than holes. Indeed, Auger energy transfer to electrons is predicted to be more efficient in materials where the ratio of electron to hole effective mass is less than one, due to the larger change in momentum required by a hole for a given change in energy. ${ }^{25,26}$

A two-beam PL experiment was performed to examine these Auger quenching mechanisms. In this experiment, a chopped $632.8 \mathrm{~nm}$ HeNe laser beam $(7 \mathrm{~mW})$ was used to probe the luminescence of $\mathrm{Er}^{3+}$ centers in a field of excess carriers created by an unmodulated $514.5 \mathrm{~nm}$ argon ion laser beam $(0-100 \mathrm{~mW})$. Both the decay lifetime and change in intensity induced by the modulated $\mathrm{HeNe}$ beam were measured as a function of the Ar beam excitation power. For the GaAs:Er sample ( $p \sim 2 \times 10^{15} \mathrm{~cm}^{-3}$ at room temperature), a sharp decrease in both the $\mathrm{Er}^{3+}$ lifetime and modulated intensity was observed even at very low Ar beam powers, suggesting that Auger quenching by excess free electrons was strong. The experimental data are shown in Fig. 6, along with the results of a model developed in the following paragraphs.

The time dependence of the excited $\mathrm{Er}^{3+}$ concentration can be modeled by the following balance:

$$
\frac{d N}{d t}=\sigma J\left(N_{T}-N\right)-\frac{N}{\tau},
$$

where $N_{T}$ is the total $\mathrm{Er}^{3+}$ concentration, $N$ is the excited $\mathrm{Er}^{3+}$ concentration, $J$ is the incident photon flux, $\tau$ is the $\mathrm{Er}^{3+}$ decay lifetime, and $\sigma$ is a pseudo-cross section for excitation. The pseudo-cross section $\sigma$ is proportional to the 
capture cross section for bound exciton formation at the Errelated trap, as well as the probability that the bound exciton excites the $\mathrm{Er}^{3+}$ ion. The latter is affected by the second Auger process in which the bound exciton excites a free electron rather than the $\mathrm{Er}^{3+}$ ion. Similarly, the first Auger process, in which the excited $\mathrm{Er}^{3+}$ ion decays nonradiatively and excites a free electron, will affect the $\mathrm{Er}^{3+}$ decay lifetime. These quenching processes will reduce $\tau$ and $\sigma$ according to

$$
\tau=\frac{\tau_{0}}{1+A n} \quad \text { and } \sigma=\frac{\sigma_{0}}{1+B n},
$$

where $\tau_{0}$ and $\sigma_{0}$ are the $\mathrm{Er}^{3+}$ lifetime and excitation cross section in the absence of Auger quenching, $A$ and $B$ are constants for the two Auger quenching processes, and $n$ is the free electron concentration. $A$ and $B$ are related to the true Auger coefficients through $A=C_{A} \tau_{0}$ and $B=C_{B} \tau_{\mathrm{BE}}$, where $W_{\mathrm{BE}}=1 / \tau_{\mathrm{BE}}$ is the intrinsic energy transfer rate from the bound exciton to the $\mathrm{Er}^{3+}$ ion. Unlike $\tau_{0}, \tau_{\mathrm{BE}}$ cannot be independently determined and separated from $C_{B}$, so only the overall constant $B$ will be discussed. The reciprocal of $A$ or $B$ gives the "critical carrier concentration" where the
Auger process becomes significant, as defined by Langer. ${ }^{21}$ The free electron concentration, $n$, will consist of the equilibrium free electron concentration, $n_{0}$, and any excess photogenerated electrons, $\Delta n$. The GaAs:Er sample used in this study showed $p$-type conductivity due to carbon impurities, so $n_{0}$ can be neglected.

In the two-beam experiment, the system starts at an initial steady state with $J$ and $n$ determined by the combined $\mathrm{HeNe}$ and Ar photon fluxes. The system then decays to a new steady state when the HeNe beam is shut off. Solving Eq. (1) for this process, using the initial condition $N(0)$ $=N_{i}$ where $N_{i}$ is defined below, yields

$$
N(t)-N_{\infty}=\left(N_{i}-N_{\infty}\right) \cdot \exp \left(-t / \tau_{\text {eff }}\right),
$$

where the effective decay lifetime is

$$
\tau_{\mathrm{eff}}=\frac{\tau_{0}}{1+A \Delta n_{\mathrm{Ar}}+\frac{\sigma_{0} \tau_{0} J_{\mathrm{Ar}}}{1+B \Delta n_{\mathrm{Ar}}}} .
$$

$N_{i}$ and $N_{\infty}$ are the initial and final excited $\mathrm{Er}^{3+}$ concentrations, determined from the steady-state solutions as

$$
N_{i}=\frac{\sigma_{0} \tau_{0}\left(J_{\mathrm{Ar}}+J_{\mathrm{HeNe}}\right) N_{T}}{\left[1+A\left(\Delta n_{\mathrm{Ar}}+\Delta n_{\mathrm{HeNe}}\right)\right] \cdot\left[1+B\left(\Delta n_{\mathrm{Ar}}+\Delta n_{\mathrm{HeNe}}\right)\right]+\sigma_{0} \tau_{0}\left(J_{\mathrm{Ar}}+J_{\mathrm{HeNe}}\right)}
$$

and

$$
N_{\infty}=\frac{\sigma_{0} \tau_{0} J_{\mathrm{Ar}} N_{T}}{\left(1+A \Delta n_{\mathrm{Ar}}\right)\left(1+B \Delta n_{\mathrm{Ar}}\right)+\sigma_{0} \tau_{0} J_{\mathrm{Ar}}} .
$$

In these expressions, the Ar and HeNe subscripts refer to the photon flux or photogenerated electron concentration due to each respective laser. This solution assumes that the excess free carrier concentration reaches its new steady-state value much faster than the excited Er ion concentration. The modulated intensity measured in the experiment (normalized to the intensity observed with no Ar beam) is

$$
\frac{I}{I_{0}}=\frac{N_{i}-N_{\infty}}{N_{i} \text { with } J_{\mathrm{Ar}}=0, \Delta n_{\mathrm{Ar}}=0} .
$$

Equations (4) and (7) can be fit to the data shown in Fig. 6 if $J$ and $\Delta n$ can be related to the HeNe and Ar laser powers. The photon flux is simply proportional to the laser power, $P$

$$
J=(1-R) \frac{P}{h \nu \cdot \pi r^{2}},
$$

where $r$ is the beam radius, and $R$ is the reflectivity at the excitation wavelength. ${ }^{27}$ The photogenerated excess electron concentration is also proportional to the laser power, ${ }^{28}$ and this proportionality constant could simply be absorbed into the constants, $A$ and $B$. However, to preserve the physical meaning of these Auger constants, the proportionality constant between $\Delta n$ and $P$ can be estimated by a model devel- oped by Mettler. ${ }^{29}$ The excess electron concentration as a function of depth into the sample is given by

$$
\begin{aligned}
\Delta n(x)= & \alpha(1-R) \frac{P}{h \nu \cdot \pi r^{2}} \cdot \frac{L_{n}^{2} / D_{n}}{\left(\alpha L_{n}\right)^{2}-1} \\
& \times\left[\frac{s_{\mathrm{rv}}+\alpha L_{n}}{s_{\mathrm{rv}}+1} \exp \left(-\alpha w+\frac{w}{L_{n}}-\frac{x}{L_{n}}\right)-\exp (-\alpha x)\right],
\end{aligned}
$$

where $\alpha$ is the absorption coefficient at the excitation wavelength, $L_{n}$ is the diffusion length of the electron, $D_{n}$ is the electron diffusion constant, $w$ is the depletion width at the surface, and $s_{\mathrm{rv}}$ is a dimensionless surface recombination velocity. $L_{n}$ is given by $L_{n}=\sqrt{D_{n} \tau_{n}}$, where $\tau_{n}$ is the minority carrier lifetime. $D_{n}$ is related to the electron mobility, $\mu_{n}$, through the Einstein relation $D_{n}=\mu_{n} k T / q$. The depletion width, $w$, can be estimated from the acceptor concentration and the band bending at the surface, assuming the Fermi level is pinned at $\sim 0.6 \mathrm{eV}$ above the valence band. Estimates for $s_{\mathrm{rv}}$ and its temperature dependence are given by Mettler. $^{29}$

An average excess electron concentration can be determined by integrating Eq. (9) over the layer thickness. Thus, the proportionality constant between $\Delta n$ and $P$ is given by

$$
k \equiv \frac{\Delta n_{\text {AVERAGE }}}{P}=\frac{1}{P \cdot l} \int_{0}^{l} \Delta n(x) \cdot d x,
$$


where $l$ is the layer thickness. Using estimates of $\tau_{n}=1 \mathrm{~ns},{ }^{30}$ $\mu_{n}=3 \times 10^{4} \mathrm{~cm}^{2} / \mathrm{V} \mathrm{s},{ }^{31}$ and $w=0.6 \mu \mathrm{m}, k$ was found to be approximately $7.0 \times 10^{8} \mathrm{~cm}^{-3} / \mathrm{mW}$ for the argon beam. Thus, the average excess electron concentration was on the order of $10^{10} \mathrm{~cm}^{-3}$ at typical powers used in the two-beam experiment.

The argon laser power dependence of the modulated intensity and effective lifetime were fit by Eqs. (4) and (7), in conjunction with Eqs. (8) and (10). The results are shown in Fig. 6. It should be noted that Eq. (7) was modified to include a $y$ offset to account for background luminescence and instrumental response which prevented $I / I_{0}$ from going to zero at high Ar powers. It was found that the data were fit well using only the second Auger process, which involves the bound exciton recombination. It was unnecessary to include the first Auger process, which involves decay of the excited $\mathrm{Er}^{3+}$ ion. ${ }^{32}$ Even when including the first process, a satisfactory fit is found only when $B \gg A$ by a factor of at least 500. The intensity $y$ offset and initial lifetime, $\tau_{0}$, are easily found at the limits of the experiment, where $P_{\mathrm{Ar}}$ is large and $P_{\mathrm{Ar}}=0$, respectively. Thus, only two parameters are varied to fit both the intensity and lifetime data: $\sigma_{0}$ and $B$. These values were found to be $\sigma_{0} \approx 1.0 \times 10^{-14} \mathrm{~cm}^{2}$ and $B \approx 2.2 \times 10^{-9} \mathrm{~cm}^{3}$. It must be emphasized that the absolute value for $B$ should only be taken as a very rough, order-ofmagnitude estimate, due to the uncertainty of the electron lifetime, electron mobility, and depletion width estimates used in determining $k$. However, the quality of the fit is in fact only determined by the combined value $k B$, which does not rely upon these estimates and is known with much more accuracy. The fact that both the intensity and lifetime curves are fit reasonably well with essentially only two varied parameters supports this model.

The model developed above suggests that a strong Auger quenching process is present, in which the bound exciton excites a free electron rather than the $\mathrm{Er}^{3+}$ ion. This quenching process would explain the decrease in intensity observed in $n$-type samples. The alternate Auger quenching process, in which the excited $\mathrm{Er}^{3+}$ ion transfers its energy to a free electron, might be present, but is much less important. The $\mathrm{Er}^{3+}$ decay lifetime is $20 \%-25 \%$ less in GaAs:Er,Se than in GaAs:Er or GaAs:Er,Zn, supporting that this process does play a minor role. However, using Eq. (2), which applies when there is only one excitation beam, $A$ is roughly estimated to be $\sim 3 \times 10^{-15} \mathrm{~cm}^{3}\left(C_{A} \sim 1.5 \times 10^{-12} \mathrm{~cm}^{3} / \mathrm{s}\right)$. Consequently, $B$ is greater than $A$ by several orders of magnitude, as was suggested earlier.

It is interesting to compare the values determined for $B$, $A$, and $\sigma_{0}$ with similar values reported in the literature. Only one critical carrier concentration $\left(B^{-1}\right)$ has been reported for the Auger process involving energy transfer from the rareearth bound exciton to a free electron. Wang and Wessels found only weak quenching in $n$-type InP:Er samples, observing appreciable $\mathrm{Er}^{3+}$ emission even with electron concentrations above $10^{19} \mathrm{~cm}^{-3} \cdot{ }^{24}$ Their value of $B \approx 2$ $\times 10^{-19} \mathrm{~cm}^{3}$ is orders of magnitude smaller than the value of $B$ determined in this study. Thus, this type of Auger quenching is much stronger for the Er-2O center in GaAs, as supported by the lack of $\mathrm{Er}^{3+}$ emission in samples with electron

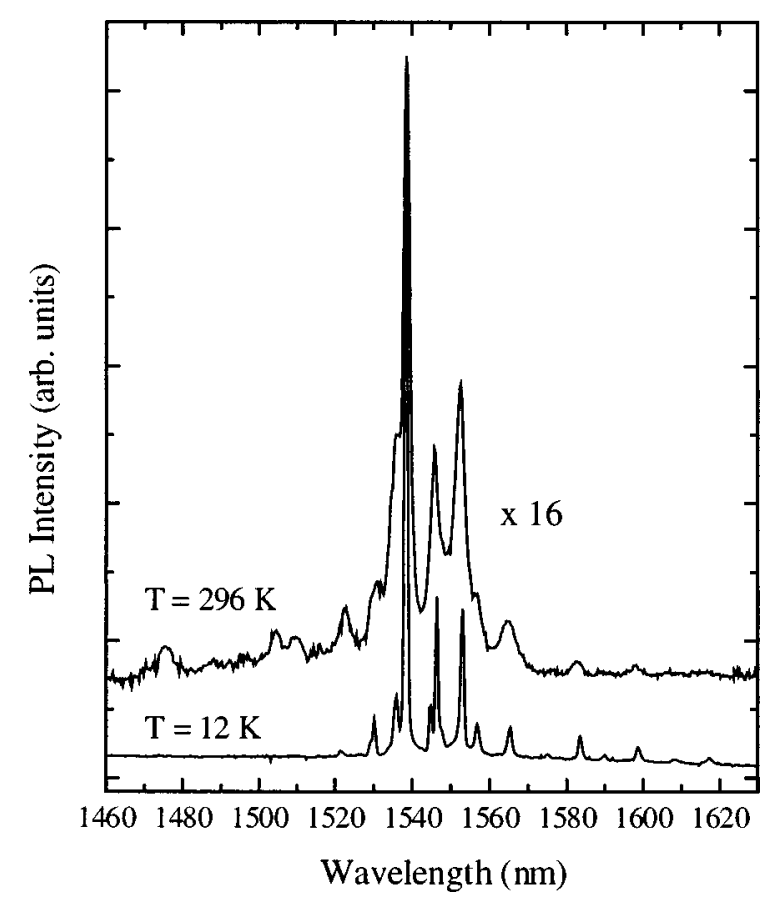

FIG. 7. PL spectra comparing the $\mathrm{Er}^{3+}$ emission of GaAs:Er at room temperature and $12 \mathrm{~K}$.

concentrations of $7 \times 10^{16} \mathrm{~cm}^{-3}$ and above. One critical carrier concentration has also been reported for the Auger process involving energy transfer from the excited rare-earth ion to a free electron $\left(A^{-1}\right)$. Langer and Van Hong found $A$ $\approx 4 \times 10^{-17} \mathrm{~cm}^{3}$ for $\mathrm{CaF}_{2}: \mathrm{Gd}^{20}$ This value is somewhat smaller than the value estimated for $A$ in the current study. Finally, Franzo et al. calculated an excitation cross section of $\sigma \approx 1 \times 10^{-16} \mathrm{~cm}^{2}$ at $77 \mathrm{~K}$ for $\mathrm{Er}^{3+}$ implanted in $\mathrm{Si}^{18}$ The cross section we have determined for the $\mathrm{Er}-2 \mathrm{O}$ center is two orders of magnitude larger than this, although a direct comparison should be viewed cautiously as Franzo et al. determined the cross section from electroluminescence measurements. In this case, the definition of the excitation cross section in terms of the carrier flux is comparable, but not strictly the same. Nonetheless, the estimates for $\sigma_{0}, A$, and $B$ in this study suggest that the $\mathrm{Er}-2 \mathrm{O}$ center in GaAs shows rather efficient excitation, but also strong Auger quenching by free electrons.

One final comment is that Eq. (7) predicts that the modulated intensity will decrease at high argon powers, even if there is no Auger quenching $(A=B=0)$. This is due to saturation of the finite $\mathrm{Er}^{3+}$ concentration by the unmodulated beam. Therefore, simply measuring the intensity decrease in a two-beam experiment is not enough to prove the existence of an Auger process, as has sometimes been claimed. Proper modeling of both the intensity and lifetime is required to support the presence of Auger quenching.

\section{Temperature dependence}

Another important issue is the temperature dependence of the $\mathrm{Er}^{3+}$ emission, because ultimately a practical device must perform at room temperature, not $12 \mathrm{~K}$. Figure 7 shows the $1.54 \mu \mathrm{m}$ emission spectra of GaAs:Er at 12 and $296 \mathrm{~K}$. 


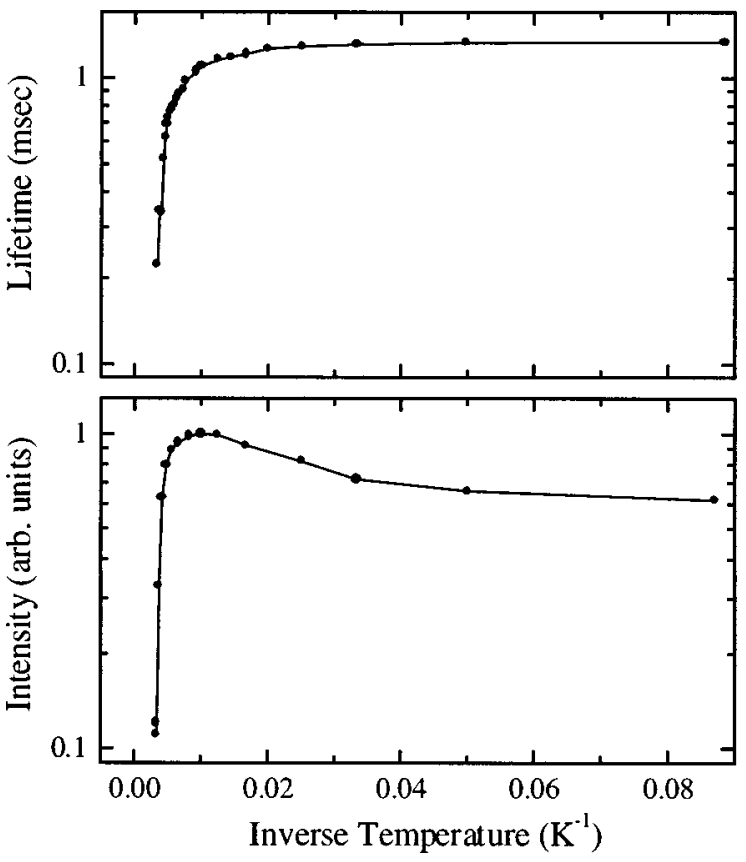

FIG. 8. Temperature dependence of the integrated intensity and main peak lifetime for the ${ }^{4} I_{13 / 2} \rightarrow{ }^{4} I_{15 / 2} \mathrm{Er}^{3+}$ emission.

As the temperature was increased to $296 \mathrm{~K}$, the integrated intensity quenched by approximately a factor of 10 . However, the main peak shifted only $2.4 \AA$ to longer wavelength over this large temperature range. This temperature stability is due to the shielded nature of the intra- $4 f$ transitions. The main peak broadened somewhat to $\sim 9 \mathrm{~cm}^{-1}$ at room temperature. Finally, several additional hot lines appeared at higher energy, resulting from transitions from higher lying states in the ${ }^{4} I_{13 / 2}$ manifold.

The thermal quenching of both the integrated intensity and main peak lifetime are shown in Arrhenius plots in Fig. 8. At higher temperatures, both the lifetime and intensity quenched with an activation energy of $290 \pm 30 \mathrm{meV}$. This value agrees well with the activation energy measured for the Er-2O center in GaAs:Er,O. ${ }^{33}$ The corresponding quenching mechanism is believed to be the process known as energy back transfer. This process is simply the reverse of the $\mathrm{Er}^{3+}$ excitation mechanism; the excited $\mathrm{Er}^{3+}$ ion decays and transfers its energy to reform the bound exciton, rather than release a photon. ${ }^{14,34}$

Upon close examination of Fig. 8, it can be seen that the intensity passed through a maximum and actually decreased at the lowest temperatures. This may indicate freezing out of free carriers, which are needed for $\mathrm{Er}^{3+}$ excitation and emission. Figure 9 shows this effect in more detail, giving the temperature dependence of the normalized intensity for different excitation powers. At higher excitation powers, the decrease in intensity at low temperatures was reduced. A similar effect was observed in the intensity of the donorbound exciton peak of the band edge luminescence, as shown in Fig. 10. These observations are consistent with free carriers freezing into a relatively shallow trap, removing their availability for $\mathrm{Er}^{3+}$ excitation or exciton recombination. Higher excitation powers saturate this trap and provide

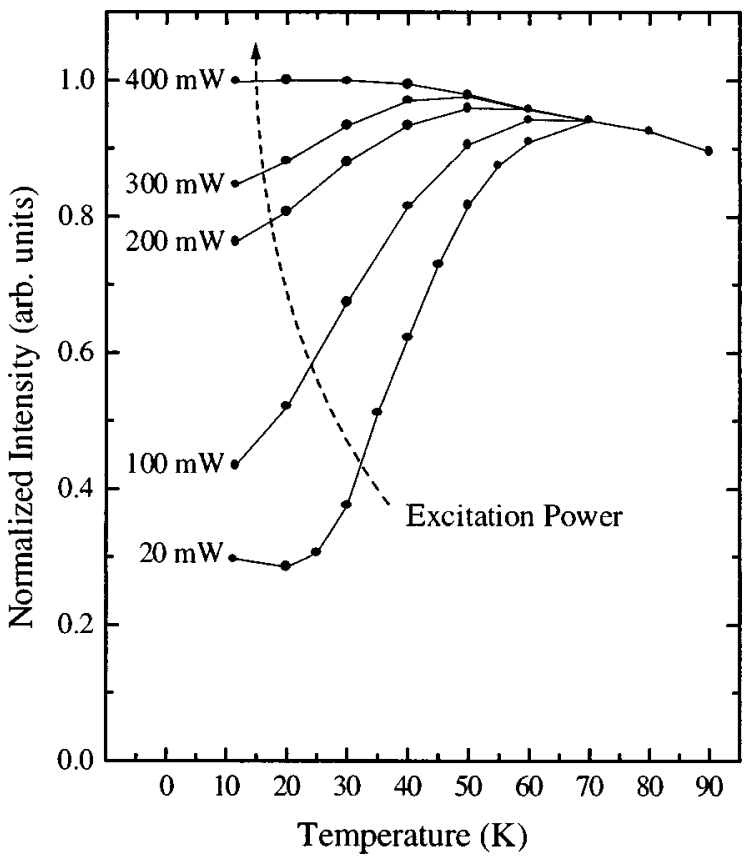

FIG. 9. $\mathrm{Er}^{3+}$ emission intensity as a function of temperature and excitation power. To better show the effect of carrier freeze-out, the curves have been normalized to meet around $70 \mathrm{~K}$, above which they show the same temperature dependence.

excess free carriers, reducing the importance of this effect.

The identity of this shallow trap is unknown at this time. Attempts to estimate the trap depth from Fermi statistics have been inconclusive, because the temperature dependence of the carrier concentration is not directly known, only the emission intensity. The freeze-out process could be the trapping of carriers into some new Er-related reservoir-type state. Or more likely, this process could be as mundane as the recapture of holes onto ionized shallow acceptors. Trap-

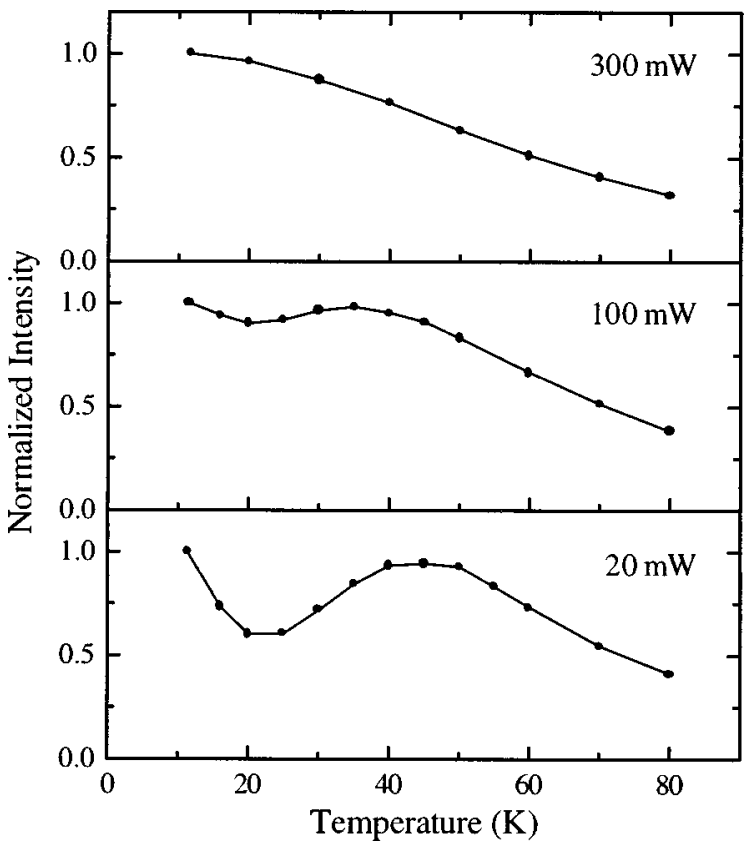

FIG. 10. Temperature and excitation power dependence of the donor-bound exciton (DX) emission of GaAs:Er. 
ping by shallow acceptors might be expected to reduce $\mathrm{Er}^{3+}$ luminescence, as it is believed that rare-earth excitation does not occur through neutral donor-acceptor pair recombination. ${ }^{35}$ Furthermore, as discussed earlier, excess free holes may be important for driving the formation of bound excitons and overcoming bound exciton thermalization. In this case, the freezing out of holes onto shallow acceptors would have a detrimental effect upon $\mathrm{Er}^{3+}$ excitation and luminescence. Future temperature-dependent Hall measurements (TDH) will help identify the nature of this shallow trap.

\section{SUMMARY}

We have investigated the characteristics of the ${ }^{4} I_{13 / 2}$ $\rightarrow{ }^{4} I_{15 / 2} \mathrm{Er}^{3+}$ emission in GaAs:Er doped with a new pyrazole and pyridine-based Er source, tris(3,5-di-tertbutylpyrazolato)bis(4-tert-butylpyridine)erbium(III). Although the concentration of $\mathrm{Er}$ incorporated into these samples was fairly low, the $\mathrm{Er}^{3+}$ emission was significantly stronger and sharper than in previous GaAs:Er samples doped with cyclopentadienyl-based Er sources. The efficient luminescence was associated with the $\mathrm{Er}-2 \mathrm{O}$ center, formed with unintentional oxygen impurities. Because of the encouraging emission characteristics in these samples despite the low Er concentration, our future work will attempt to preferentially form this $\mathrm{Er}-2 \mathrm{O}$ center in higher concentrations by using an intentional oxygen source. The source molecule could either be $\mathrm{O}_{2}$, as used by Takahei et al., ${ }^{7}$ or $\left(\mathrm{C}_{2} \mathrm{H}_{5}\right)_{2} \mathrm{AlOC}_{2} \mathrm{H}_{5}$, which has been used to dope GaAs:O. ${ }^{36}$

The $\mathrm{Er}^{3+}$ emission was greatly reduced in $n$-type samples, whereas the emission remained strong in $p$-type samples. This effect was ascribed to two possibilities, both of which might be important. First, the free hole concentration may be very important to the $\mathrm{Er}^{3+}$ excitation efficiency, in that excess holes are needed to overcome thermalization of the Er-bound exciton through hole emission. Second, measurements of the emission lifetime and modulated intensity in a two-beam experiment supported the presence of a strong Auger quenching mechanism which involves free electrons. A model was developed and compared to experimental results, indicating strong Auger energy transfer from the bound exciton to a free electron. Auger energy transfer from the excited $\mathrm{Er}^{3+}$ ion to a free electron was not found to be significant.

The temperature dependence of the $\mathrm{Er}^{3+}$ emission was also examined. At high temperatures, thermal quenching due to energy back transfer was observed. At the lowest temperatures, the samples also showed a decrease in intensity. This effect was attributed to the freeze-out of carriers onto a relatively shallow trap which could be related to either Er or shallow acceptors.

In conclusion, we have observed several effects that show the importance of the interaction between Er centers and free carriers. In order to fully exploit the properties of these materials, an important goal will be a thorough understanding of these interactions.

\section{ACKNOWLEDGMENTS}

The authors gratefully acknowledge financial support from the National Science Foundation and a UW-WARF fellowship, as well as the U.S. Army Research Office. The authors would also like to thank MCNC Analytical Labs who attempted the SIMS analysis.

${ }^{1}$ Rare Earth Doped Semiconductors, Materials Research Society Symposium Proceedings 1993, edited by G. S. Pomrenke, P. B. Klein, and D. W. Langer (Materials Research Society, Pittsburgh, 1993), Vol. 301.

${ }^{2}$ Rare Earth Doped Semiconductors II, Materials Research Society Symposium Proceedings 1996, edited by S. Coffa, A. Polman, and R. N. Schwartz (Materials Research Society, Pittsburgh, 1996), Vol. 422.

${ }^{3}$ J. M. Redwing, D. C. Gordon, B. A. Vaartstra, S. S. Lau, and T. F. Kuech, J. Appl. Phys. 76, 1585 (1994).

${ }^{4}$ T. D. Culp, U. Hömmerich, J. M. Redwing, T. F. Kuech, and K. L. Bray, J. Appl. Phys. 82, 368 (1997)

${ }_{5}^{5}$ D. Pfeiffer, L. M. Liable-Sands, A. L. Rheingold, M. J. Heeg, and C. H. Winter (in preparation).

${ }^{6}$ J. G. Cederberg, T. D. Culp, B. Beig, D. Pfeiffer, C. H. Winter, K. L. Bray, and T. F. Kuech, J. Appl. Phys. (submitted).

${ }^{7}$ K. Takahei, R. A. Hogg, and A. Taguchi, Mater. Res. Soc. Symp. Proc. 422, 267 (1996).

${ }^{8}$ K. Takahei, A. Taguchi, Y. Horikoshi, and J. Nakata, J. Appl. Phys. 76, 4332 (1994).

${ }^{9}$ D. Haase, A. Dörnen, K. Takahei, and A. Taguchi, Mater. Res. Soc. Symp. Proc. 422, 179 (1996).

${ }^{10}$ R. A. Hogg, K. Takahei, A. Taguchi, and Y. Horikoshi, Mater. Res. Soc. Symp. Proc. 422, 167 (1996).

${ }^{11}$ K. Takahei and A. Taguchi, J. Appl. Phys. 77, 1735 (1995).

${ }^{12}$ K. Takahei and A. Taguchi, J. Appl. Phys. 78, 5614 (1995).

${ }^{13}$ H. Nakagome, K. Uwai, and K. Takahei, Appl. Phys. Lett. 53, 1726 (1988).

${ }^{14}$ K. Takahei, A. Taguchi, H. Nakagome, K. Uwai, and P. S. Whitney, J. Appl. Phys. 66, 4941 (1989).

${ }^{15}$ K. Thonke, K. Pressel, G. Bohnert, A. Stapor, J. Weber, M. Moser, A. Molassioti, A. Hangleiter, and F. Scholz, Semicond. Sci. Technol. 5, 1124 (1990).

${ }^{16}$ K. Takarabe, T. Mizushima, S. Minomura, A. Taguchi, and K. Takahei, Mater. Sci. Forum 196-201, 645 (1995).

${ }^{17}$ J. Michel, J. Palm, F. Gan, F. Y. G. Ren, B. Zheng, S. T. Dunham, and L. C. Kimerling, Mater. Sci. Forum 196-201, 585 (1995).

${ }^{18}$ G. Franzo, F. Priolo, C. Spinella, and S. Coffa, J. Appl. Phys. 81, 2784 (1997)

${ }^{19}$ T. Gregorkiewicz, I. Tsimperidis, C. A. J. Ammerlaan, F. P. Widdershoven, and N. A. Sobolev, Mater. Res. Soc. Symp. Proc. 422, 207 (1996).

${ }^{20}$ J. M. Langer and L. Van Hong, J. Phys. C 17, L923 (1984)

${ }^{21}$ J. M. Langer, J. Lumin. 40, 41, 589 (1988).

${ }^{22}$ T. Benyattou, D. Seghier, G. Guillot, R. Moncorge, P. Galtier, and M. N. Charasse, Appl. Phys. Lett. 58, 2132 (1991).

${ }^{23}$ X. M. Fang, Y. Li, and D. W. Langer, J. Appl. Phys. 74, 6990 (1993).

${ }^{24}$ X. Z. Wang and B. W. Wessels, Mater. Sci. Forum 196-201, 657 (1995).

${ }^{25}$ J. S. Blakemore, in Semiconductor Statistics (Dover, New York, 1987), Chap. 6.

${ }^{26}$ P. T. Landsberg, in Recombination in Semiconductors (Cambridge University Press, Cambridge, 1991), Chap. 5.

${ }^{27}$ An alternate approach would be to consider $J$ as the photogenerated carrier flux through the layer, rather than directly as the absorbed photon flux. An average magnitude of the carrier flux could then be calculated by averaging $\left|D_{n} d \Delta n / d x\right|$ across the layer, where $\Delta n$ is proportional to the laser power and given by Eq. (9). However, it should be noted that the cross-section, $\sigma_{0}$, will be different in each case, defined for excitation either based upon the incident photon flux or based upon the carrier flux across the layer. Using this alternate definition, $\sigma_{0}$ is found to be 3.8 $\times 10^{-12} \mathrm{~cm}^{2} . B$ is not affected by the definition of $J$.

${ }^{28}$ Bimolecular recombination, where $\Delta n$ is proportional to $P^{1 / 2}$, is not expected to be dominant under the conditions of this experiment, where the photogenerated electron concentration is small $\left(\Delta n \sim 10^{10} \mathrm{~cm}^{-3}\right)$. See Ref. 25.

${ }^{29}$ K. Mettler, Appl. Phys. 12, 75 (1977). 
${ }^{30}$ S. Gupta, S. Sethi, and P. K. Bhattacharya, Appl. Phys. Lett. 62, 1128 (1993). Lifetime estimate was roughly adjusted for temperature.

${ }^{31} \mathrm{~S}$. Adachi, in GaAs and Related Materials (World Scientific, London, 1994), Chap. 14.

${ }^{32}$ Note that although it seems counterintuitive to what is observed in traditional one-beam PL experiments, the lifetime measured in this two-beam experiment will decrease at very low Ar laser powers even when Auger quenching of the excited $\mathrm{Er}^{3+}$ ion is not included. In Eq. (4), $\tau_{\text {eff }}$ can be seen to depend upon $B$ and the $\mathrm{Ar}$ laser power as well as $A$.
${ }^{33}$ A. Taguchi and K. Takahei, J. Appl. Phys. 79, 4330 (1996).

${ }^{34}$ A. Taguchi, H. Nakagome, and K. Takahei, Appl. Phys. Lett. 60, 965 (1992).

${ }^{35}$ T. Gregorkiewicz, B. J. Heijmink Liesert, I. Tsimperidis, I. de MaatGersdorf, C. A. J. Ammerlaan, M. Godlewski, and F. Scholz, Mater. Res. Soc. Symp. Proc. 301, 239 (1993).

${ }^{36}$ J. W. Huang, D. F. Gaines, T. F. Kuech, R. M. Potemski, and F. Cardone, J. Electron. Mater. 23, 659 (1994). 